\title{
Robust implementation under alternative information structures
}

\section{Luis C. Corchon *, Ignacio Ortuño-Ortin}

\author{
anstituto Valenciano de Investigaciones Económicas and Departamento de Fundamentos del Análisis \\ Económico, Universidad de Alicante, Alicante, Spain \\ ${ }^{\mathrm{b}}$ Departamento de Fundamentos del Análisis Económico, Universidad de Alicante, Alicante, Spain
}

Received December 1993; revised April 1994

\section{Abstract}

In this paper we consider a model in which agents have complete information about their neighbors and, possibly, incomplete information about the rest of the environment. We consider two different informational frameworks. In the first, agents do not have priors about the relevant characteristics in the rest of the environment. In the second, agents are supposed to have priors about the unknown characteristics. We present a mechanism which implenients any social choice correspondence satisfying monotonicity and no veto power in both informational settings for every possible prior thus requiring little knowledge from the point of view of the designer of the information possessed by agents about the environment.

Keywords: Nash Implementation; Incomplete information; Local information

JEL classification: CF2, DF1, D82

\footnotetext{
The authors wish to thank J. Canals, B. Chakravorty, P. Chander, C. Herrero, G. Orosel, D. Schmeidler, W. Thomson, W. Trockel, F. Vega, A. Villar, T. Yamato and two anonymous referees for helpful comments. The usual disclaimer applies. The first author acknowledges financial support from the Institució Valenciana d'Estudies i Investigació; I.V.I.E and DGICYT under projects PB/88-0289 and $\mathrm{PB} / 91-0756$. The second author acknowledges financial support from DGICYT under project PB/90-0156. A previous version of the paper was written when authors visited (May 1991) the Institute of Mathematical Economics (Bielefeld) to which authors are grateful.

* Corresponding author.
} 


\section{Introduction}

The Theory of Implementation studies the feasibility of achieving social goals when taking properly into account agents' incentives, i.e. the possibility of reconciliating utopia and self-interested behavior. Usually, an agent's behavior is modeled according to some game-theoretical concept which is suited to an informational framework. Social goals are described by a mapping, called Social Choice Correspondence (SCC in the sequel), which associates to each environment in a certain class a set of feasible alternatives. This set is interpreted as the socially optimal alternatives for a given environment. A Mechanism (sometimes called a Game Form) is a description of the language in which agents communicate and the consequences of the messages (strategies) they send. A mechanism is said to implement a given SCC if, for any environment in the domain of this correspondence, there are equilibrium messages (in some game-theoretical sense) and the consequences of these messages coincide with the alternative prescribed by the SCC.

Most papers in Implementation Theory assume that the designer knows the equilibrium concept, i.e. how agents behave. The purpose of this paper is to investigate the consequences of assuming that the mechanism should work, at least partially, regardless of the structure of information in the environment and therefore, it should implement the SCC for two equilibrium concepts rather than for a single one. The idea behind this, is that when designing a mechanism, the planner does not know the structure of information. Therefore the mechanism should be robust to the different possibilities that might arise. In this sense, our paper is akin to the work on Double Implementation which requires a mechanism to implement a given SCC in both Nash and Strong equilibria (see Maskin (1979 and 1985), Schmeidler (1980), Corchon-Wilkie (1991), Tadenuma-Thomson (1993) and Suh (1993)) or in Nash and undominated Nash equilibria (see Jackson (1989), Jackson-Palfrey-Srivastava (1990) and Yamato (1991a)). Our concept of Robust Implementation is conceived in the same spirit, but applied to the problem of information. We require a mechanism to implement a SCC under two different informational structures: ignorance and risk. Therefore, we might say that our mechanism doubly implements a SCC but keeping in mind that in our case the different equilibrium concepts refer to different informational set-ups.

A typical result in the Theory of Implementation says that, given a kind of rational behavior (implied by the structure of information), some SCC can or can not be implemented in a certain range of environments. Thus, if agents use dominant strategies, implementation of acceptable SCC is essentially impossible if the domain of SCC is large enough (Hurwicz (1972), Gibbard (1973), Satterthwaite (1975), see also Ledyard-Roberts (1974)). However, implementation of acceptable SCC is possible, under certain conditions, if the equilibrium concept is Nash (Maskin (1977), see also Repullo (1987)). Moreover these conditions can be adapted to the case in which agents are assumed to be Bayesian (see Postlewaite- 
Schmeidler (1986), Palfrey-Srivastava (1989) and Jackson (1991)). All these results suggest a trade-off between the information possessed by an agent about other agents - none in the case of dominant strategies, a common prior in the Bayesian framework and complete information in the case of Nash - and the possibility of implementing a satisfactory SCC.

In this paper we will consider the implementation problem when the information possessed by agents is variable. In order to give structure to the problem we will make an additional-assumption: throughout the paper we will assume that agents have complete information about a part of the environment. In other words, the environment is composed of islands, with a population of at least three agents each. Every agent has complete knowledge about the preferences of other people on the same island. This fact is also common knowledge to the agents and the designer. Examples of islands include families, groups of friends, firms in the same industry, individuals in a task force, departments in a university, etc. With respect to the information about the rest of the environment, we will assume two different settings.

1. Ignorance. Agents only know the set of possible types of agents outside their islands, as opposed to their actual types. Moreover, priors on types are meaningless. In this framework, the equilibrium strategy for an agent must be the best reply to what the rest of the agents on the island play, and to any possible message sent by agents outside her island when they follow their equilibrium strategies. The last requirement resembles dominant strategies, the difference being that a strategy is dominant if it is a best reply regardless of how other players behave, and in our case, the strategy must be a best reply no matter what other players (outside the island) are like. This equilibrium concept has been used in the literature (without our assumption about islands) under the name of Uniform Nash Equilibrium (see d'Aspremont-Gerard-Varet (1979) and Matsushima (1988)).

2. Risk. In this framework, our equilibrium concept is the usual Bayesian Equilibrium introduced by Harsanyi (1967), with the aforementioned condition that players have complete information inside islands. The difference here from the usual notion of Bayesian implementation is that we assume that the designer does not know the exact prior. This implies that our mechanism implements a given SCC in Bayesian equilibrium for any possible prior. Thus the same mechanism implements under complete information, i.e. in Nash equilibrium. Therefore our results here are, in this sense, stronger than the standard results of Bayesian implementation.

We define a mechanism as Robust relative to a SCC, if it implements this correspondence in Uniform Nash Equilibrium and Bayesian Equilibrium for any possible prior (i.e. with the designer being ignorant of the actual prior). This notion attempts to capture the fact that the mechanism should be flexible enough to cope with different informational settings and that changes in the structure of information do not therefore imply a change in the mechanism. It must be remarked that our paper addresses a different problem from the one studied by 
Saijo (1988) and McKelvey (1989). There, it was shown how the strategy space for Nash Implementation - under complete information - can be reduced. In contrast, we will consider implementation problems where agents only need to have complete information about their own island. A more satisfactory concept of robustness would allow for the possibility that islands might be populated by agents who have priors about some subset of agents outside the island and at the same time are ignorant about other agents outside the island.

Our main result is that there is a Robust mechanism which implements any SCC satisfying the well-known conditions of Monotonicity and Non-Veto Power introduced by Maskin in the framework of Nash Implementation. ${ }^{1}$ Thus, given our assumption about islands, the fact that information is or is not complete makes no essential difference from the point of view of Implementation. Therefore our results can be understood as a generalization of those obtained by Maskin under complete information. The cost of this generalization is the common knowledge assumption on islands.

We remark that in this paper we do not identify in general necessary and sufficient conditions for robust implementation. However, notice that since robust implementation implies Nash implementation all necessary conditions of the latter are necessary conditions of the former as well. In particular monotonicity or the conditions identified in Moore-Repullo (1990) as necessary conditions of Nash implementation are necessary conditions of robust implementation. Moreover, in economic environments, no veto power is vacuously satisfied and thus monotonicity is a necessary and sufficient condition for robust implementation.

An alternative motivation for our approach is that the theory of Nash implementation has been criticized for the following reason. On the one hand, the outcome function is not continuous on messages (at least when the general implementation problem is studied). On the other hand, exact complete information is required. Therefore a slight mistake in other people's characteristics might entail alternatives far away from those selected by the SCC. Our mechanism however only requires exact complete information on a, usually small, subset of agents. Thus, our mechanism can be regarded as introducing some robustness on Nash implementation.

It should be remarked that the paper does not make any progress in other important topics such as the reduction of strategy spaces (even though our strategy spaces are not much larger than those in Williams (1986), Saijo (1988) and McKelvey (1989)), the avoidance of modulo games (see Jackson (1988)), the consideration of continuous and 'realistic' mechanisms and the possibility of agents forming coalitions or playing mixed strategies. A careful study of these points would obscure considerably the main concern of the model.

\footnotetext{
${ }^{1}$ It is not difficult to show that in our model -private values and non-exclusive informationmonotonicity implies Bayesian monotonicity.
} 
The rest of the paper goes as follows. The next section explains the basic model and our main assumptions. Section 3 is devoted to proving our results and, finally, section 4 gathers our conclusions together.

\section{The model}

In this section we present our basic framework. Let $N=\{1, \ldots, n\}$ be the finite - set of agents. Let $T_{i}$ be the (finite but greater than one) set of attributes of player $i$ and $t_{i}$ an element of $T_{i}$. We assume that $t_{i}$ embodies all relevant information about agent $i$. Let $T=X_{i=1}^{n} T_{i}$ be the set of states. A state $t \in T$ provides a complete description of information and preferences of all agents. Let $\mathscr{A}$ stand for the set of social alternatives and let $\mathscr{F}: T \rightarrow \mathscr{A}$ be the social choice correspondence (SCC in the sequel). This mapping is assumed to embody the social objectives.

We now describe the informational framework. We will assume throughout the paper that there is a partition of $N, G=\left(G_{1}, \ldots, G_{s}\right)$ with \# $G_{i}>2, i=1, \ldots, s$ such that each agent in a given element of $G$ has complete information about all the characteristics of any agent in this element of the partition, and this is common knowledge for all agents in the partition. Notice that this partition need not be unique. We may think of the environment as being composed of islands in the terminology introduced by Lucas (1972) (but notice that here, any island is composed of at least three agents). Therefore in our model information is not exclusive. Also, each agent is assumed to know the information contained in sets $N, T, \mathscr{A}$, and the SCC $\mathscr{F}$. This information is assumed to be common knowledge. Let $G_{k}$ be a typical element of $G$ and $i$ a typical element of $G_{k}$. Let $t_{\{i\}}$ be the information of $i \in G_{k}$ about the attributes of agents on her island, i.e. $t_{i i}=$ $\left(t_{j}, \ldots, t_{p}\right)$ where $j, \ldots, p$ are all the agents in $G_{k}$. We write $T_{[i]}=T_{j} \times \ldots \times T_{p}$. Also let us denote the attributes of all agents outside $G_{k}$ by $t_{-\{i\}}$ and $T_{-\{i\}}=X_{j \notin G_{k}}$ $T_{j}$. We will say that $t_{\{i\}}$ is the type of agent $i$.

With respect to the information possessed by an agent about other islands, we will consider two set-ups. In the first one, agents will be assumed to act under ignorance, i.e. they do not assign probabilities to the occurrence of states of the world (which are the types of agents living on other islands). In the second one, agents are considered to be Bayesian, i.e. they assign probabilities to the states of the world.

Each agent has a utility function $u_{i}: \mathscr{A} \times T_{i} \rightarrow \mathbb{R}$, i.e. we assume private values (it is not difficult to extend our results to the case in which the utility function depends on the type of the agent). Let us denote by $L\left(a, t_{i}\right)$ the elements of $\mathscr{A}$ which are not preferred to $a \in \mathscr{A}$ according to the utility function $u_{i}\left(, t_{i}\right)$, i.e. the lower contour set of agent $i$ relative to her utility function. Let us define $a_{i}^{M}\left(t_{i}\right) \subseteq \mathscr{A}$ as the set of maximal elements of $\mathscr{A}$ according to $u_{i}\left(, t_{i}\right)$. It is assumed that this set is not empty for all possible attributes (see Jackson (1991) p. 
469 and Palfrey-Srivastava (1991) p. 482 for a similar assumption). This assumption holds if, for instance, preferences are continuous, and the feasible set is compact.

We now define the strategic elements. A Mechanism (Game Form) is a pair $\left(\mathscr{K}, g\right.$ ) where $g: \mathscr{H} \rightarrow \mathscr{A}$ and $\mathscr{H}=X_{i=1}^{n} \mathscr{M}_{i} . g$ is the outcome function and $\mathscr{M}_{i}$ is the message space of agent $i$. A strategy for agent $i$ is a function $s_{i}: T_{\{i\}} \rightarrow \mathscr{M}_{i}$. We write $s$ as the vector of strategies for all agents, i.e. $s=\left(s_{1}, \ldots, s_{n}\right)$. Also $s_{\{i\}}=\left(s_{j}, \ldots, s_{p}\right)$ is the tuple of strategies of all agents inside $G_{k}$, and $s_{-\{i\}}$ the tuple of strategies of all agents outside $G_{k}$.

In the first informational setting, we assume that agents act in complete ignorance of the characteristics of any agent outside her group. Therefore each agent will play 'Nash' against agents within her group and uniform Nash against any agent outside her group. Formally

Definition 1. $s^{*}=\left(s_{1}^{*}, \ldots, \mathrm{s}_{n}^{*}\right)$ is a Uniform Nash Equilibrium with Complete Local Information for the state $t=\left(t_{1}, \ldots, t_{n}\right)$ if $\forall i=1, \ldots, n$

$$
\begin{aligned}
& u_{i}\left(g\left(s_{\{i\}}^{*}\left(t_{[i\}}\right), s_{-\{i\}}^{*}\left(t_{-\{i\}}\right)\right), t_{i}\right) \geq u_{i}\left(g\left(m_{i}, s_{\{i\} / i}^{*}\left(t_{\{i\}}\right), s_{-\{i\}}^{*}\left(t_{-\{i\}}\right), t_{i}\right),\right. \\
& \forall t_{-\{i\}} \in T_{-\{i\}}, \quad \forall m_{i} \in M_{i} .
\end{aligned}
$$

Notice that the equilibrium strategy of any agent must maximize her utility for any possible message recommended by the mapping $s_{-\{i\}}^{*}\left(T_{-(i)}\right)$ for all agents outside $G_{k}$. Let $U N E(t, \mathscr{M}, g)$ be the set of Uniform Nash Equilibria with Complete Local Information (or Uniform Nash Equilibria for short) for the Game Form $(\mathscr{K}, g)$ when the state is $t$.

Definition 2. $(\mathscr{K}, g)$ implements the SCC $\mathscr{F}$ in $U N E$ if $\forall t \in T$

(a) $\forall a \in \mathscr{F}(t), \exists s^{*} \in U N E(t, \mathscr{M}, g)$ such that $a=g\left(s^{*}\right)$.

(b) If $s^{*} \in U N E(t, \mathscr{M}, g)$ then $g\left(s^{*}\right) \in \mathscr{F}(t)$.

We will say that $\mathscr{F}$ is implementable in Uniform Nash Equilibrium if there is a mechanism implementing $\mathscr{F}$ when agents behave according to Definition 1.

Our definition of a Uniform Nash equilibrium does not pay attention to the case in which agents have priors about the occurrence of states of the world. In order to deal with this case let us introduce a new informational setting. Agents have a common prior distribution $p(t)$ which is assumed to be common knowledge. Let $P$ be the set of all admissible common prior distributions $p$ on $T$. In order to keep matters simple, we will assume that either there is complete information or $p(t)>0 \forall t \in T$. In accordance with the previous ideas, we can define the conditional probability distribution for agents on island $G_{k}, q_{\{i\}}\left(t_{-\{i\}} \mid t_{\{i\}}\right)$, by

$$
q_{\{i\}}\left(t_{-\{i\}} \mid t_{\{i\}}\right)=\frac{p\left(t_{-\{i\}}, t_{\{i\}}\right)}{p_{\{i\}}\left(t_{\{i\}}\right)},
$$


where

$$
p_{\{i\}}\left(t_{\{i\}}\right)=\sum_{t_{-\{i\}} \in T_{-\{i\}}} p\left(t_{-\{i\}}, t_{[i]}\right) .
$$

We also assume that agent $i$ 's preferences on risky outcomes can be represented by a von Neumann-Morgenstern utility function.

Definition 3. $s^{*} \equiv\left(s_{1}^{*}, \ldots, s_{n}^{*}\right)$ is a Bayesian Equilibrium with Complete Local Information if, for a given state of the world $t$ and common prior distribution $p(\cdot)$ and for all $i \in N$, we have

$$
\begin{aligned}
& \sum_{t_{-i i\}} \in T_{-\{i\}}} q_{\{i\}}\left(t_{-\{i\}} \mid t_{\{i\}}\right) u_{i}\left(g\left(s_{(i)}^{*}\left(t_{\{i\}}\right), s_{-\{i\}}^{*}\left(t_{-\{i\}}\right)\right), t_{i}\right) \geq \\
& \sum_{t_{-\{i\}} \in T_{-(i)}} q_{\{i\}}\left(t_{-\{i\}} \mid t_{(i)}\right) u_{i}\left(g\left(m_{i}, s_{\{i \backslash \backslash i}^{*}\left(t_{[i\}}\right), s_{-\{i\}}^{*}\left(t_{-\{i\}}\right)\right), t_{i}\right), \quad \forall m_{i} \in \mathscr{M}_{i} .
\end{aligned}
$$

This is the usual definition of Bayesian equilibrium for the information structure given above. Let $B(\mathscr{M}, g, p, t)$ be the set of Bayesian Equilibria with Complete Local Information (Bayesian Equilibrium for short) for the Game form $(\mathscr{A}, g$ ) when the state of the world is $t$ and the common prior distribution is $p$.

We remind the reader that the standard Bayesian implementation approach assumes that the designer of the mechanism knows the common prior distribution. In our model, the center does not need to know $p$. However, it must know the partition $G$ which seems to us to be a less demanding informational requirement, at least in some cases.

Definition 4. The Game Form $(\mathscr{M}, g)$ implements the $S C C \mathscr{F}$ in Bayesian Equilibrium with Complete Local Information if for all $t \in T, p \in P$

(1) $\forall a \in \mathscr{F}(t), \exists s^{*} \in B(\mathscr{M}, g, p, t)$ such that $g\left(s^{*}\right)=a$

(2) If $s^{*} \in B(\mathscr{M}, g, p, t)$, then $g\left(s^{*}(t)\right) \in \mathscr{F}(t)$.

Notice that the complete information model is a particular case of the Bayesian framework where $q$ 's are either zero or one. Thus, Bayesian implementation implies Nash implementation. Finally, we come to the main notion of the paper.

Definition 5. Let $\mathscr{F}$ be a $S C C$. The mechanism $(\mathscr{K}, g)$ is Robust relative to $\mathscr{F}$ if it implements $\mathscr{F}$ in Uniform Nash Equilibrium and Bayesian Equilibrium.

The idea behind Definition 5 is that a mechanism is robust relative to $\mathscr{F}$ (in short, robust) if it implements $\mathscr{F}$ irrespective of the information on the environ- 
ment. Therefore the designer does not need to have much knowledge of the information that agents possess on the environment. Moreover, if agents acquire more information or change their priors, the proposed mechanism still implements $F$.

Before we present our main result we will define two concepts which will play an important role in our paper. Both are standard in the theory of Nash implementation.

A SCC $\mathscr{F}$ satisfies monotonicity if for all $t, t^{\prime} \in T$ and $a \in \mathscr{F}\left(t^{\prime}\right)$ we have $L_{i}\left(a, t_{i}^{\prime}\right) \subset L_{i}\left(a, t_{i}\right)$ for all $i \in N$ only if $a \in \mathscr{F}(t)$.

A SCC $\$$ satisfies no veto power if for all $i \in N, t \in T$ and $a \in T$ and $a \in A$ we have $L_{i}\left(a, t_{j}\right)=A$ for all $j \neq i$ only if $a \in \mathscr{F}(t)$.

It should be pointed out that an implication of monotonicity is that if $t \neq t^{\prime}$ but $u_{i}\left(, t_{i}\right)=u_{i}\left(, t_{i}^{\prime}\right) \forall i$, then $\mathscr{F}(t)=\mathscr{F}\left(t^{\prime}\right)$. In this sense, the set of alternatives recommended by $\mathscr{F}$ depends only on preferences and not on the information structure. Since Robust Implementation implies Nash implementation and monotonicity is a necessary condition of Nash Implementation, it is also a necessary condition of Robust Implementation. Our main result is that monotonicity (plus no veto power) is sufficient for Robust Implementation.

Theorem 1. Any SCC which satisfies Monotonicity and No Veto Power can be implemented by a robust mechanism relative to $\mathscr{F}$.

Firstly, we will present the mechanism. The next section will then be devoted to proving that this mechanism implements in Uniform Nash and Bayesian Equilibria any SCC satisfying monotonicity and no veto power.

Let the message space for agent $\mathrm{i}$ be given by

$$
\mathscr{M}_{i}=T_{\{i\}} \times F \times \mathscr{A} \times \mathscr{F}(T) \times \mathbb{N} \times \mathscr{A},
$$

where $F$ is the set of all selections of $\mathscr{F}, \mathscr{F}(T)$ is the range of the $S C C \mathscr{F}$ and $\mathbb{N}$ is the set of natural numbers. We write an element of $\mathscr{K}_{i}$ in the following way

$$
m_{i}=\left(t_{i}^{i}, t_{i}^{j}, \cdots t_{i}^{p}, f_{i}, a_{i}, c_{i}, n_{i}, b_{i}\right),
$$

Thus, $t_{i}^{j}$ will be the 'report' on agent $j$ 's attributes given by agent $i$. We will write $t_{i}^{(i)}=\left(t_{i}^{i}, t_{i}^{j}, \cdots \cdot t_{i}^{p}\right)$, i.e. $t_{i}^{(i)}$ is the information provided by $i$ about the attributes of the people on her island. We now define the outcome function by way of the following three rules

Rule 1. If $m=\left(m_{1}, \ldots, m_{n}\right)$ is such that $t_{i}^{j}=t_{j}^{j}$ for all $i, j \in G_{k}$ and $k=1, \ldots, s$, and there exists $f \in F$ such that $f_{i}=f$ for all $i$, then $g(m)=f\left(t_{1}^{1}, \ldots, t_{n}^{n}\right)$.

Rule 2. There exists a unique group $k$ and there is a selection $f \in F$ such that for all $G_{m} \neq G_{k}$ we have $t_{s}^{r}=t_{r}^{r}$ and $f_{s}=f_{r}=f$ for all $s, r \in G_{m}$ and for the group $G_{k}$ 
there exists a unique $i \in G_{k}$ such that $\left(t_{i}^{(i)}, f_{i}\right) \neq\left(t_{j}^{(i)}, f_{j}\right)$ with $\left(t_{j}^{(i)}, f_{j}\right)=\left(t_{h}^{(i)}, f_{h}\right)$ and $f_{j}=f \forall j, h \in G_{k}, j, h \neq i$. Then

$$
g(m)=\left|\begin{array}{c}
a_{i}, \quad \text { if } a_{i} \in L\left(c_{i}, t_{j}^{i}\right) \text { and } c_{i}=f\left(t_{1}^{1}, \ldots, t_{j}^{i}, t_{j}^{j}, \ldots, t_{n}^{n}\right) \\
\text { where } j \in G_{k} \\
f\left(t_{1}^{1}, \ldots, t_{j}^{i}, t_{j}^{j}, \ldots, t_{n}^{n}\right), \quad \text { otherwise. } \\
.
\end{array}\right|
$$

Rule 3: If Rules 1 or 2 do not apply, then $g(m)=b_{i}$, where $i=\max \left\{j: n_{j} \geq n_{s}\right.$, for all $s \in N$ )

When Rule 1 applies we say that there exists complete agreement on the reports. Whenever Rule 2 applies we say that there exists a unique disagreement and agent $i$ is the dissident. If Rule 3 applies it is said that there are at least two dissidents.

Rule 1 says that if there is total consistency on the reports on states and the selection of $\mathscr{F}$, the mechanism chooses the alternative given by the selection $f$ using the reported states.

Rule 2 takes care of the case whereby all agents but one send consistent reports on states. In this case the mechanism will choose the alternative $a_{i}$ given by the 'dissident' whenever (1) $c_{i}$ coincides with the alternative recommended by the chosen selection $f$ and the states reported by all other agents (2) this alternative belongs to the lower contour set of $c_{i}$ using the states reported by all other agents on $i$. If $a_{i}$ does not satisfy (1) and (2), the mechanism chooses the alternative recommended by the selection $f$ when states are those reported by all agents but the 'dissident'.

In all other cases, i.e. when there is more than one 'dissident' Rule 3 applies. These rules allow for some possible interpretation of $a_{i}, c_{i}$ and $b_{i}$ as a choice, a guess and a best alternative in $\mathscr{A}$ respectively.

\section{The proof of the theorem}

This section will be devoted to proving Theorem 1. We will divide the proof into two Propositions. These Propositions will show that the proposed mechanism implements a SCC in Bayesian and Uniform Nash Equilibria respectively.

\section{Proposition 1.}

If the Social Choice Function $\mathscr{F}$ satisfies Monotonicity and No Veto Power, then the above Game Form $(\mathscr{M}, g$ ) implements $\mathscr{F}$ in Bayesian Equilibrium.

Proof: We will present the proof for the case of $p(t)>0 \forall t$. The case of complete information is totally analogous and it is omitted. 
(a) Firstly, we show that part 1 of Definition 4 is satisfied by $(\mathscr{K}, g)$, i.e., $\forall t \in T, \forall p \in P$, and for any $a \in \mathscr{F}(t)$ there exists $s^{*} \in B(\mathscr{M}, g, p, t)$ such that $g\left(s^{*}(t)\right)=a$. Choose any selection $f \in F$ such that $f(t)=a$ and consider for any agent $i$ the 'truth-telling' strategy $s_{i}^{*}: T_{(i)} \rightarrow \mathscr{K}$ given by $s_{i}^{*}\left(t_{(i)}\right)=\left(t_{i i\}}, f, a_{i}, c_{i}\right.$, $n_{i}, b_{i}$ ) where $a_{i}, c_{i}, n_{i}, b_{i}$ are arbitrary choices and $t_{\{i\}}$ is the true type of agent $i$. We now show that $s^{*} \in B(\mathscr{M}, g, p, t)$. If all agents follow these strategies, Rule 1 applies and the outcome is $g\left(s^{*}(t)\right)=f(t)$. Suppose that agent $i$ chooses $m_{i}=\left(t_{i}^{(i)}, f_{i}^{\prime}, a_{i}^{\prime}, c_{i}^{\prime}, n_{i}^{\prime}, b_{i}^{\prime}\right)$ instead of $s_{i}^{*}\left(t_{(i)}\right)$, with $\left(t_{i}^{(i)}, f_{i}^{\prime}\right) \neq\left(t_{(i)}, f\right)$. Thus, Rule 2 applies, and the outcome is either $a_{i}^{\prime}$ or $f(t)$. In both cases the outcome cannot be preferred to $g\left(s^{*}(t)\right)$

(b) Secondly, we show that $(\mathscr{K}, g$ ) satisfies (2) in Definition 4. Now three cases must be considered.

(i) $g\left(s^{*}(t)\right)$ is given by Rule 1, i.e., $s^{*}(t)$ yields a complete agreement. In this case any agent $i$ could have chosen a message $m_{i}=\left(\bar{t}_{i}^{(i)}, \bar{f}_{i}, \bar{a}_{i}, \bar{c}_{i}, \vec{n}_{i}, \bar{b}_{i}\right)$ instead of $s_{i}^{*}\left(t_{\{i\}}\right)=\left(t^{(i)}, f_{i}, a_{i}, c_{i}, n_{i}, b_{i}\right)$, where $\left(t^{(i)}, f_{i}\right) \neq\left(t_{i}^{(i)}, f_{i}\right), \bar{a}_{i} \in L\left(\bar{c}_{i}, t_{i}^{i}\right), \bar{n}_{i}=\hat{n}$ $\left(s^{*}\right), \bar{b}_{i} \in a_{i}^{M}\left(t_{i}\right)$ and $\bar{c}_{i}=f_{i}\left(t^{*}\right)$, where $t^{*}$ are the reports on the states associated to $s^{*}(t)$ and $\hat{n}\left(s^{*}\right)$ is a natural number greater than any number recommended by the equilibrium strategy for any agent and any state of the world $\left(\hat{n}\left(s^{*}\right)\right.$ is well defined because the finiteness of $N$ and $T$ ). We want to know the reason why agent $i$ did not choose message $m_{i}$. For all $\bar{t}_{-(i)}$ such that $s^{*}\left(t_{(i)}, \bar{t}_{-\{i\}}\right)$ produces at least one dissident, the announcing of $m_{i}$ will take the mechanism to Rule 3 and the outcome will be $g\left(s_{i}^{*}(t), m_{i}\right) \in a_{i}^{M}\left(t_{i}\right)$. For all the cases $t_{-\{i\}}^{\prime}$ such that $s^{*}\left(t_{i j}, t_{-\{i\}}^{\prime}\right)$ produces complete agreement, the announcing of $m_{i}$ yields the outcome $g\left(s_{-\{i\}}^{*}\left(t_{-\{i\}}^{\prime}\right), \quad s_{\{i\}}^{*}\left(t_{i i\}}\right)\right)$ whenever $\bar{c}_{i} \neq f_{i}\left(t^{1}, \ldots, t_{j}^{i}, t_{1}^{\mathrm{j}}, \ldots, t_{n}^{n}\right)$ where $\left(t_{1}^{t}, \ldots, t_{j}^{i}, t_{j}^{j}, \ldots, t_{n}^{n}\right)$ are the report on types associated to $s^{*}\left(t_{-(i\}}^{\prime}, t_{\{-i\}}\right)$, i.e. the reports on types given by all agents but agent $i$, and $a_{i}$ whenever $\bar{c}_{i}=f_{i}\left(t_{1}^{1}, \ldots, t_{j}^{i}\right.$, $t_{j}^{j}, \ldots, t_{n}^{n}$ ). Thus, the reason for which she did not choose $m_{i}$ must be that

$$
\vec{a}_{i} \in L\left(\bar{c}_{i}, t_{i}^{i}\right) \Rightarrow \bar{a}_{i} \in L\left(\bar{c}_{i}, t_{i}\right) .
$$

This implication holds for all agents. Therefore by monotonicity of $\mathscr{F}$ and since $\bar{c}_{i}=f\left(t^{*}\right)$ for all $i$, then $f\left(t^{*}\right) \in \mathscr{F}(t)$ and $g\left(s^{*}(t)\right) \in \mathscr{F}(t)$.

(ii) $g\left(s^{*}(t)\right)$ is given by Rule 2, i.e. $g\left(s^{*}(t)\right)$ yields a unique dissident. Let agent $i \in G_{k}$ be the dissident. In this case, it is clear that for all $j \in G_{k}, j \neq i$, we have $g\left(s^{*}(t)\right) \in a_{j}^{M}\left(t_{j}\right)$. To see this note that agent $j$ can always choose message $m_{j}=\left(\bar{t}_{j}^{(j)}, \bar{f}_{j}, \bar{a}_{j}, \bar{c}_{j}, \bar{n}_{j}, \bar{b}_{j}\right)$ such that $\left(\bar{t}_{j}^{(j)}, \bar{f}_{j}\right) \neq\left(t_{i}^{[i]}, f_{i}\right),\left(\bar{t}_{j}^{(j)}, \bar{f}_{j}\right) \neq\left(t_{l}^{(j)}, f_{l}\right), l \in G_{k}$, $l \neq i, b_{j} \in a_{j}^{M}\left(t_{j}\right)$ and $\bar{n}_{j}=\hat{n}\left(s^{*}\right)$. By the choice of $m_{j}$ agent $j$ takes the outcome from Rule 2 to Rule 3, and in this case she can get her best alternative. Now we show that the same must be true for agents outside $G_{k}$, i.e., $g\left(s^{*}(t)\right) \in a_{u}^{M}\left(t_{u}\right)$ $\forall u \notin G_{k}$. Take an agent $\mathrm{U} \in G_{r}$. Suppose that $g\left(s^{*}(t)\right) \notin a_{u}^{M}\left(t^{\prime}\right.$. Agent $u$ could have chosen, instead of the message $s_{u}^{*}\left(t_{(u)}\right)=\left(t_{u}^{(u)}, f_{u}, a_{u}, c_{u}, n_{u}, b_{u}\right)$, the message $m_{u}=\left(\bar{t}_{u}^{(u)}, \bar{f}_{u}, \bar{a}_{u}, \bar{c}_{u}, \bar{n}_{u}, \bar{b}_{u}\right)$ where $\left(t_{u}^{(u)}, f_{u}\right) \neq\left(\bar{t}_{u}^{(u)}, \bar{f}_{u}\right), \bar{a}_{u}=\bar{c}_{u}, \bar{n}_{u}=\hat{n}\left(s^{*}\right)$ and $\vec{b}_{u} \in a_{u}^{M}\left(t_{u}\right)$. For all $t_{-\{u\}}$ such that $s^{*}\left(t_{\{u\}}, t_{-(u)}\right)$, is given by rule $1, m_{u}$ and 
$s_{u}^{*}\left(t_{(u)}\right)$ yield the same outcome. To see this, observe that for these cases, by the choice of $m_{u}$, agent $u$ can force the mechanism to go to Rule 2 (and she becomes the dissident). Since $a_{u}=c_{u}$ his dissidence is, however, a vacuous one. For all $t_{-\{u\}}$ such that $s^{*}\left(t_{\{u\}}, t_{-\{u\}}\right)$ is given by Rules 2 or 3 message $m_{u}$ is such that $g\left(s_{-u}^{*}(t), m_{u}\right)$ is always given by Rule 3 and since $\bar{n}_{u}=\hat{n}\left(s^{*}\right)$ we have $g\left(s_{u}^{* *}(t), m_{u}\right)=\bar{b}_{u} \in a_{u}^{M(}\left(t_{u}\right)$. Therefore for some $t_{-(u)}$, the message $m_{u}$ and $s_{u}^{*}\left(\bar{t}_{\{u\}}\right)$ yield the same outcome and for the rest of $t_{-\{u\}}$, message $m_{u}$ yields an outcome which is strictly preferred by agent $u$ to the outcome achieved by announcing $s_{u}^{*}\left(t_{\{u\}}\right)$ [observe that since we are in the case where $g\left(s^{*}(t)\right)$ is given by Rule 2, there always exists such a $t_{-\{u\}}$ for which $m_{u}$ is strictly preferred to $s_{u}^{*}$ ]. Thus, regardless of the conditional probability function, $m_{u}$ is strictly preferred to $s_{u}^{*}\left(t_{\{u\}}\right)$, which is a contradiction. Therefore, $g\left(s^{*}(t)\right) \in a_{u}^{M}\left(t_{u}\right)$ for all $u \notin G_{k}$. Thus, we have that $g\left(s^{*}(t)\right) \in a_{j}^{M}\left(t_{j}\right), \forall j \neq i$, and by No Veto Power of $\mathscr{F}$ we have that $g\left(s^{*}(t)\right) \in \mathscr{F}(t)$.

(iii) $g\left(s^{*}(t)\right)$ is given by Rule 3 , i.e. there are at least two dissidents. In this case it is clear that all agents obtain the best outcome, i.e., $g\left(s^{*}(t)\right) \in a_{i}^{M}\left(t_{i}\right)$. Then by No Veto Power of $\mathscr{F}, g\left(s^{*}(t)\right) \in \mathscr{F}(t)$.

\section{Proposition 2.}

If the Social Choice Correspondence $F$ satisfies Monotonicity and No veto Power, then the above Game form $(\mathscr{M}, g)$ implements $\mathscr{F}$ in Uniform Nash Equilibrium with Complete Local Information.

Proof. The first part of this Proposition (i.e. $\forall a \in \mathscr{F}(t), \exists s^{*} \in U N E(t, \mathscr{M}, g)$ such that $\left.a=g\left(s^{*}\right)\right)$ can be done in the same way as in Proposition 1 , using 'truth-telling' strategies. The second part of the proof (i.e. If $s^{*} \in U N E(t, \mathscr{M}, g)$ then $\left.g\left(s^{*}\right) \in \mathscr{F}(t)\right)$, can be proved by noting that $\mathrm{UNE}(t, \mathscr{K}, g) \subseteq B(\mathscr{K}, g, p, t)$ $\forall p, t$. Therefore $g(\mathrm{UNE}(t, \mathscr{A}, g)) \subseteq g(B(\mathscr{A}, g, p, t))$. By Proposition 1, $g(B(\mathscr{K}, g, p, t)) \subseteq \mathscr{F}(t)$ and this proves the claim.

\section{Conclusions}

In this paper we have shown that under some assumptions - including private values and that a most preferred element in the set of social alternatives exists for every agent - the existence of complete information inside islands guarantees the existence of a Robust Mechanism relative to any SCC satisfying the well-known conditions of monotonicity and no-veto power. Therefore, under our assumptions, the sufficient conditions for implementation in Nash Equilibrium turn out to be sufficient for implementation under Uniform Nash and Bayesian Equilibria as well. This means that if No-Veto Power is guaranteed, we can achieve the same alternatives under complete and incomplete information. This is basically due to 
the fact that in our case, information is not exclusive (see Palfrey-Srivastava (1986) and Blume-Easley (1990)). ${ }^{2}$

Our result contrasts with that obtained by Ledyard (1978) about the impossibility of implementing with Bayesian Equilibrium for every prior a large class of SCC, and with the so-called 'incentive compatibility approach to organization design' in which the choice of the mechanism depends crucially on prior beliefs (see Ledyard (1987) p. 149 and also Mookherjee-Reichelstein (1989), p. 1). In this approach first best alternatives can not usually be achieved (see HarrisTownsend (1981) pp. 36-7, 53-4 and 58 for a discussion on ex-ante vs ex-post efficiency and prior-free mechanisms). Therefore, the simplifications introduced in this paper must be judged with regard to the positive results which are achieved. We believe that the approach we have started in this paper can provide a new avenue of research by identifying conditions under which incomplete information reduces to complete information. Some progress in this direction has been made by Yamato (1991b).

We finally remark on an interesting feature of our mechanism. If $\mathscr{F}$ is a function and the set of maximal elements is always a singleton, it can be shown that the strategies which are a Bayesian Equilibrium are (ex post) a Nash Equilibrium. Therefore no agent will ever regret her choice.

\section{References}

D'Aspremont, C. and Gerard-Varet, A. 1979, Incentives and incomplete information, Journal of Public Economics 11, 25-45.

Blume, L. and Easley, D. 1990, Implementation of Walrasian expectations equilibria, Journal of Economic Theory 51, 207-227.

Corchon, L. and Wilkie, S., 1991, Doubly implementing the ratio correspondence by means of a market mechanism, discussion paper, U. of Alicante.

Gibbard, A., 1973, Manipulation of voting schemes: A general result, Econometrica 41, 587-602.

Harris, M. and Townsend, R., 1981, Resource allocation under asymmetric information, Econometrica $49,33-64$.

Harsanyi, J.C., 1968; Games with incomplete information played by bayesian players, Management Science $14,159-189,320-334$ and 486-502.

Hurwicz, L., 1972, On informationally decentralized systems, Decision and Organization: A volume in honor of Jacob Marschak, ed. R. Radner and C.B. McGuire, 297-336. Amsterdam: North-Holland Publishing Co.

Jackson, M.O., 1989, Implementation in undominated strategies: A look at bounded mechanisms. Forthcoming in the Review of Economic Studies.

Jackson, M.O. 1991, Bayesian implementation, Econometrica 59, 461-77.

\footnotetext{
${ }^{2}$ The readers may wonder if implementation under some kind of Dominant-Nash strategies (instead of Uniform-Nash) can occur in our informational setting. However it is easy to find an example disproving this conjecture.
} 
Jackson, M.O. and Palfrey, T. and Srivastava, S., 1990, Undominated Nash implementation in bounded mechanisms. Games and Economic Behavior (forthcoming).

Ledyard, J. and Roberts, J., 1974, On the incentive problem with public goods, Discussion Paper 116, Center for Mathematical Studies in Economics and Management Science, Northwestern University.

Ledyard, J., 1978, Incomplete information and incentive compatibility Journal of Economic Theory 18, $171-89$.

Ledyard, J., 1987, Incentive compatibility in Allocation, Information and Markets ed. by J. Eatwell, M. Milgate and $\mathbf{P}$. Newman.

Lucas, R.E., 1972, Expectation and the neutrality of money, Journal of Economic Theory 4, 103-124.

Maskin, E., 1977, Nash equilibrium and welfare optimality, Department of Economics, Massachusetts Institute of Technology. Mimeo.

Maskin, E., 1979, Incentives schemes immune to group manipulation. Mimeo.

Maskin, E., 1985, The theory of implementation in Nash equilibrium: a survey in Social Goals and Social Organization ed. by L. Hurwicz, D. Schmeidler and H. Sonnenschein. London, Cambridge University Press.

Matsushima, H., 1988, A new approach to the implementation problem, Journal of Economic Theory $45,128-144$.

McKelvey, R.D., 1989, Game forms for Nash implementation of general social choice correspondences, Social Choice and Welfare 6, 139-156.

Moore, J. and Repullo, R., 1990, Nash implementation: a full characterization, Econometrica 56, 1191-1220.

Mookherjee, D. and Reichelstein, S. 1989, Dominant strategy implementation of Bayesian incentive compatible rules. Mimeo, Graduate School of Business, Stanford University.

Palfrey, T.R. 1990, Implementation in Bayesian equilibrium invited paper to the 6th. World Congress of the Econometric Society.

Palfrey, T.R. and Srivastava, S., 1986, Private information in large economies, Journal of Economic Theory, 39, 34-58.

Palfrey, T.R. and Srivastava, S., 1989, Implementation with incomplete information in exchange economies, Econometrica 57, No. 1, 115-134

Palfrey, T.R. and Srivastava, S., 1991. Nash implementation using undominated strategies, Econometrica $59,479-501$.

Postlewaite, A. and Schmeidler, D. 1986, Implementation in differential information economies, Journal of Economic Theory 39, 14-33.

Repullo, R. 1987 A simple proof of Maskin's theorem on Nash implementation, Social Choice and Welfare 4, 39-41.

Saijo, T., 1988, Strategy space reduction in Maskin's theorem: Sufficient conditions for Nash implementation, Econometrica 56, No. 3, 693-700.

Satterthwaite, M. 1985, strategy-proofness and Arrow's conditions: Existence and correspondence theorems for voting procedures and social welfare functions, Journal of Economic Theory 10 , 187-217.

Schmeidler, D., 1980, Walrasian analysis via strategic outcome functions. Econometrica 48, 1585-1593.

Suh, S-C., 1993, Doubly implementing the equitable and efficient solutions. Mimeo, University of Rochester, July.

Tadenuma, K. and Thomson, W., 1993, Games of fair division. Mimeo, University of Rochester.

Williams, S.R. 1986, Realization and Nash implementation: Two aspects of mechanism design, Econometrica 54, No. 1, 139-151.

Yamato, T., 1991a Nash implementation and double implementation: Equivalence theorems. Mimeo, University of Rochester, August.

Yamato, T., 1991b, Equivalence of Nash implementability and Robust implementability with incomplete information. Mimeo, University of Rochester, October. 